\title{
Extended Dominating Set in Ad Hoc Networks Using Cooperative Communication ${ }^{\star}$
}

\author{
Jie Wu, Mihaela Cardei, Fei Dai, and Shuhui Yang \\ Department of Computer Science and Engineering, \\ Florida Atlantic University, Boca Raton, FL 33431
}

\begin{abstract}
We propose a notion of extended dominating set whereby each node in an ad hoc network is covered by either a dominating neighbor or several 2-hop dominating neighbors. This work is motivated by cooperative communication in ad hoc networks where transmitting independent copies of a packet generates diversity and combats the effects of fading. In this paper we propose several efficient heuristic algorithms for constructing a small extended dominating set.
\end{abstract}

\section{Introduction}

Dominating set (DS) has been widely used in ad hoc networks. A set is dominating if every node in the network is either in the set or a neighbor of a node in the set. When a DS is connected (i.e., its induced graph is connected), it is denoted as CDS. The problem of finding the minimum DS and minimum CDS is NP-complete. Many heuristic protocols have been proposed to find a minimal DS or CDS [1] [2] [3].

We propose a notion of extended dominating set based on cooperative communication (CC) [4]. CC makes single-antenna nodes in a multi-user scenario share their antennas to create a virtual multiple-input multiple-output (MIMO) system. CC can potentially combine the following two advantages: (1) the power savings provided by multi-hopping, and (2) the spatial diversity provided by the antennas of separate mobile nodes. In CC, transmitting independent copies of a packet generates diversity and combats the effects of fading. In this way, $k$ copies of the same packet can potentially reach a receiver outside the normal transmission range without increasing transmit power. Under the CC model, a DS is called an extended dominating set (EDS) if, for every node in the network, it is in the set, it has a neighbor in the set, or it has $k$ 2-hop neighbors in the set. In Fig. 11(a), $\{u, v, w\}$ forms a CDS. If using CC, and $k=2$, node $x$ is covered by two 2-hop neighbors, $u$ and $v$. Then, $w$ can be withdrawn and $\{u, v\}$ forms an EDS. Since the set is connected, it is also called an extended connected dominating set (ECDS). Later, we will define weakly connected EDS (EWCDS). In EWCDS, the broadcast will be successful for at least one source in EDS; whereas in the ECDS the broadcast will be successful for any source in the EDS. In Fig.11(b), $\{u, v, x\}$ forms an EWCDS for $k=2$ since $x$ can retrieve the complete packet when either $u$ or $v$ is the source, while neither $u$ nor $v$ can when $x$ is the source.

\footnotetext{
* This work was supported in part by NSF grants ANI 0073736, CCR 0329741, CNS 0422762, CNS 0434533, and EIA 0130806. Contact address: jie@ cse.fau.edu.
} 


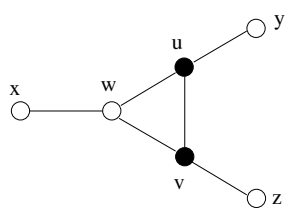

(a)

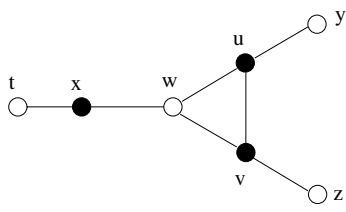

(b)

Fig. 1. (a) A sample with CDS: $\{u, v, w\}$ and ECDS: $\{u, v\}$. (b) EWCDS: $\{x, u, v\}$.

Wu and Lou [5] classified CDS formation methods into global, quasi-global, quasilocal, and local depending on the amount of information each node has, and the complexity of both message and time to determine a CDS. This paper focuses on some non-trivial extensions of various methods for ECDS/EWCDS and proposes (1) global solutions for EWCDS, (2) quasi-global solutions for EWCDS, (3) quasi-local solutions for EDS and ECDS, and (4) local solutions for EDS and ECDS. For more technical details, theorem proofs and simulation results, readers are refereed to [6].

\section{Extended Dominating Set}

Given a set $V$ of points in a 2D space, a normal transmission range $r$, and a CC range $r^{\prime}$, we define a graph with vertex set $V$ and an arc from vertex $v$ to vertex $u$ iff the Euclidean distance, $d(v, u)$, is no more than $r$. In addition, we define a quasi-arc from vertex $v$ to vertex $u$ iff $r<d(v, u) \leq r^{\prime}$. When $r^{\prime}=2 r$, the corresponding graph can be approximated by a single unit disk graph, where a quasi-arc exists between any two vertices (called quasi neighbors) that are separated by two hops.

Definition 1. A subset of nodes is an EDS if every node is (a) in the subset, $(b)$ a regular neighbor of a node in the subset, or (c) a quasi neighbor of $k$ nodes in the subset.

Definition 2. An EDS is strongly connected under the CC model (denoted as ECDS) if for any node $u$ in the set sending a packet, the packet should be fully received by all other nodes eventually. Only nodes with a fully received packet (including u) are able to forward the packet once.

If the connectivity condition holds for at least a particular node $u$, it is called weakly connected (EWCDS). It is known that DS and CDS problems in unit disk graphs are NP-complete. We proved that EDS, ECDS, and EWCDS problems are NP-complete.

The ECDS/EWCDS can be used as a virtual backbone under the CC model. Such a backbone can support an efficient broadcast process and reduce searching space. Unlike broadcasting using regular DS, the source node may need a relay node (not in the EDS) to forward the packet to a node in the EDS; otherwise, only the nodes in the EDS need to forward the packet. 


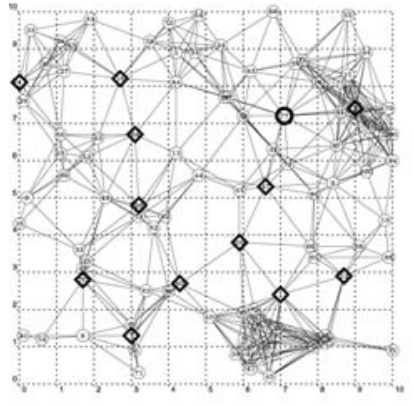

(a) EWCDS by E-MCDS, size $=13$, source is 77 .

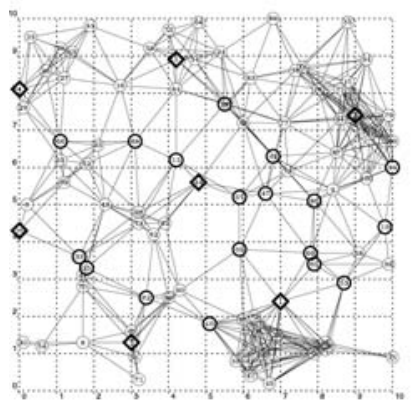

(c) ECDS by E-Cluster-LMST, size $=25$.

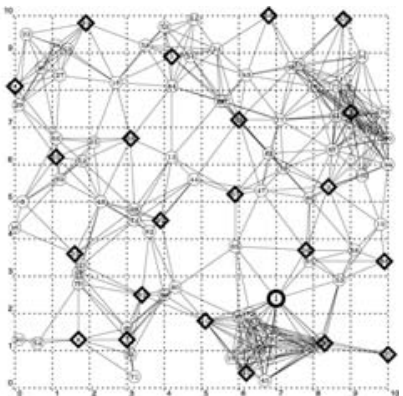

(b) EWCDS by E-AWF, size $=23$, source is 1 .

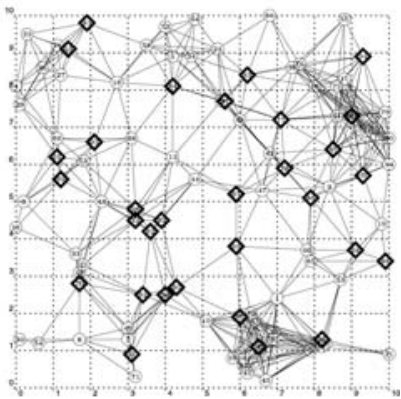

(d) ECDS by E-Rule $K$, size $=31$.

Fig. 2. Sample ECDS or EWCDS in an ad hoc network with 100 nodes

\section{Heuristic Solutions}

Global solutions for EWCDS. We consider a centralized greedy solution called extended MCDS (E-MCDS), based on Guha and Khuller's MCDS [1]. MCDS "grows" a tree from a selected root until all nodes are covered. Non-leaf nodes form a CDS. We introduce the notion of contribution here: Each forward node contributes 1 to all its neighbors and $1 / k$ to all quasi neighbors. The reception ratio of a node is the combined contribution of its forward (quasi) neighbors. The algorithm is to find a minimum EWCDS so that all other nodes are reachable (i.e., each node has a reception ratio of at least 1). To ensure a constant approximation ratio, we employ a mutual exclusion rule which uses the concept of independent set. Fig. 2 (a) shows the EWCDS generated by the E-MCDS in a random, 100-node connected graph.

Quasi-global solutions for EWCDS. We extend the AWF algorithm [2] for CDS. AWF contains topology sorting, sequential clustering, and gateway designation procedures. In our extended AWF algorithm for EWCDS (E-AWF), we modify the gateway designation procedure to use an extended gateway designation approach, thus the set of selected nodes becomes an EWCDS, and the algorithm has a constant approximation ratio. Fig. 2 (b) shows the EWCDS generated by E-AWF.

Quasi-local solutions for EDS and ECDS. We use the clustering approach as the solution for EDS and ECDS. By a quasi-local solution, we mean the solution completes 
with a high probability in a small number of rounds with an occasional large number of rounds for completion. The clustering algorithm contains the selection of clusterheads and gateways. In our extended clustering approach (E-Clustering), each node operates on its 2-hop neighborhood. When a clusterhead is chosen, it dose not only contribute 1 to the coverage of its neighbors, but also $1 / k$ to its quasi neighbors. To extend EDS to ECDS, we use an extension of the local minimum spanning tree (LMST) algorithm [7] to select gateways, whereby the 1-hop neighborhood includes the current clusterhead, all clusterheads within 5 hops, and their pairwise minimum "virtual path" in terms of hop count. In this way, each pair of neighboring clusterheads has a virtual link and LMST can be applied. The EDS generated by extended clustering and gateway nodes together forms an ECDS that has a constant approximation ratio. In Fig.2 (c), the clusterheads are noted by diamonds, and the gateways by bold circles.

Local Solutions for EDS and ECDS. In local backbone construction, each node maintains only 2-hop information and performs: (1) Dai and Wu's pruning rule [3] (Rule $K$ ) for constructing a CDS, and (2) an aggressive pruning rule to remove nodes from the CDS while still maintaining local coverage and connectivity. We develop the extended Rule $K$ (E-Rule $K$ ), which uses 2-hop information, including the markers of all 2-hop neighbors. A marked node $u$ can be unmarked if all its 2-hop neighbors, regular and quasi, can be covered (including contribution cumulation) by other marked nodes with higher priority (noted as $C$ ) in the neighborhood, and the corresponding condition is called the coverage condition. The set derived by the pruning rule based on the coverage condition forms an EDS. To ensure connectivity, we require $C$ to be connected under the $\mathrm{CC}$ model, the connectivity condition. We call $C$ an extended component if it is strongly connected (based on Definition 2). A pruning rule that meets coverage and connectivity conditions preserves an ECDS with the expected size $O(1) \cdot\left|E C D S_{o p t}\right|$, where $E C D S_{\text {opt }}$ is an optimal solution to the ECDS problem. Fig. 2 (d) shows the ECDS generated by the E-Rule $K$.

\section{References}

1. Guha, S., Khuller, S.: Approximation algorithms for connected dominating sets. Algorithmica 20 (1998) 374387

2. Alzoubi, K.M., Wan, P.J., Frieder, O.: Distributed heuristics for connected dominating set in wireless ad hoc networks. Journal of Communications and Networks 4 (2002) 2229

3. Dai, F., Wu, J.: An extended localized algorithm for connected dominating set formation in ad hoc wireless networks. IEEE Transaction on Parallel and Distributed Systems 15 (2004) 908920

4. Nosratinia, A., Hunter, T.E., Hedayat, A.: Cooperative communication in wireless networks. IEEE on Communications 42 (2004) 7480

5. Wu, J., Lou, W.: Forward node set based broadcast in clustered mobile ad hoc networks. Wireless Communications and Mobile Computing 3 (2003) 141154

6. Wu, J., Cardei, M., Dai, F., Yang, S.: Extended dominating set and its applications in ad hoc networks using cooperative communication. (submitted for publication)

7. Li, N., Hou, J., Sha, L.: Design and analysis of an MST-based topology control algorithm. In: Proceedings of INFOCOM. (2003) 